\title{
Correlation of microstructure and constitutive behaviour of sintered silver particles via nanoindentation
}

\author{
Xu Long ${ }^{\mathrm{a}, *}$, Bo Hu ${ }^{\mathrm{b}}$, Yihui Feng ${ }^{\mathrm{c}}$, Chao Chang ${ }^{\mathrm{d}}$, Mingyu Li ${ }^{\mathrm{b}, *}$ \\ a School of Mechanics, Civil Engineering and Architecture, Northwestern Polytechnical University, Xi'an 710072, China \\ ${ }^{\mathrm{b}}$ Harbin Institute of Technology Shenzhen Graduate School, Materials Science and Engineering, Shenzhen Key Laboratory of Advanced Materials, Shenzhen 518055, \\ China \\ ' State Key Laboratory of Nonlinear Mechanics, Institute of Mechanics, Chinese Academy of Sciences, Beijing 100190, China \\ d School of Applied Science, Taiyuan University of Science and Technology, Taiyuan 030024, China
}

\section{A R T I C L E I N F O}

\section{Keywords:}

Silver nanoparticles

Silver microparticles

Nanoindentation

Microstructure

Constitutive behaviour

\begin{abstract}
A B S T R A C T
During the sintering process, a three-dimensional network-like porous structure forms as the consequences of atom diffusion and neck formation among silver particles. In this paper, two representative types of pressureless sintered silver particles, i.e., silver nanoparticles (AgNP) and silver microparticles (AgMP), are investigated to reveal the intrinsic correlation between microstructure and constitutive behaviour. The heterogeneous microstructure is found to result in distinguishing differences in terms of morphology and thermal stability. The difference of sintering mechanisms between these two types of silver pastes are observed by a scanning electron microscope and discussed. Regarding thermal stability, the effect of particle size and shape on the sintering temperature and mass loss are evaluated using the thermogravimetric analysis and the differential scanning calorimetry. The microstructural effect on the mechanical behaviour of both sintered AgNP and AgMP samples is measured by performing nanoindentations with a Berkovich indenter penetrated up to $2000 \mathrm{~nm}$ with different indentation strain rates. During indentation, Young's modulus and hardness are measured as the functions of penetration depth. By solving dimensionless equations, the parameters of a power-law constitutive model are obtained analytically based on the integrated work done in the loading stage and the contact stiffness in the unloading stage in the nanoindentation experiments. The correlation between microstructure and constitutive behaviour is elucidated by the comparison of sintered AgNP and AgMP but further investigations related to the size and shape effects of particles are expected.
\end{abstract}

\section{Introduction}

Die-attach materials in semiconductor devices serve as thermalmechanical connections. With the development of wide-bandgap semiconductors such as electronic devices based on the SiC technology, traditional die-attach materials including lead-free solder alloys are difficult to satisfy the demand of high heat dissipation as well as high temperature reliability [1-3]. Due to the characteristics of low-temperature sintering and high-temperature working, the paste with silver particles is becoming one of the potential die-attach materials of electronic packaging structure of high-density microsystems [4]. As the strain and the induced stress in the die-attach layer usually occur because of the mismatch of thermal expansion coefficients (CTE) between different material layers, the constitutive behaviour of the sintered silver paste has to be well described with the help of experimental and numerical results prior to reliable incorporations into extensive applications. In the ex- isting publications, a unified visco-plastic Anand model was applied to study the tensile behavior and ratcheting behavior of sintered silver interconnects $[1,5]$. Creep behavior of sintered silver lap-shear joints was investigated and a constitutive relation for the steady-state creep was established by $\mathrm{Li}$ et al. [6]. In order to focus on the intrinsic local properties by ruling out the size effect of specimens, the strain rate sensitivity with indentation strain rates and penetration depths are investigated by a novel technique of multiple strain-rate jumps, and the stress exponent is also determined to indicate the deformation mechanism of creep behavior by Long et al. [7]. However, technical challenges still exist due to the lack of constitutive models for the thermal-mechanical reliability evaluation of electronic packaging structures.

The sintered silver paste is usually of porous morphology, which makes its mechanical behavior more complicated. After reviewing the existing studies, three aspects are found to be closely associated with the porosity to indicate the intrinsic porous morphology of sintered silver

\footnotetext{
* Corresponding authors.

E-mail addresses: xulong@nwpu.edu.cn (X. Long), myli@hit.edu.cn (M. Li).
} 
paste. That is, the particle size distribution in the unsintered state, the pressure and temperature during the sintering process and the pore evolution during the service condition. More importantly, the porosity induced by these three aspects is strongly related to the mechanical properties, which offers the possibility that the mechanical necessities can be optimized by tuning the relevant aspects to obtain the preferred porosity and morphology. Firstly, the particle size distribution has been reported to influence the shear strength and the porosity of sintered silver joints. Ide et al. [8] found that the shear strength of joints sintered by nanoparticles of $11 \mathrm{~nm}$ was measured to be 4 times higher than that sintered by $100 \mathrm{~nm}$ particle. Long et al. [9] evaluated the porosity based on the microstructures of sintered silver paste after different sintering conditions and the Young's modulus is determined by performing nanoindentation experiments. Zhao et al. [10] utilized a mixture of silver flakes at the micrometer scale, silver flakes at the sub-micrometer scale and silver particles at the nanometer scale to achieve the porosity of $12 \%$, which is $8 \%$ lower than that of the sintered silver paste with only nanoparticles. Secondly, it is also found that the applied pressure during the sintering process can lead to less porosity and higher shear stress [11]; nevertheless, the pressure may induce damage to some fragile components $[12,13]$. As for sintering temperature, Zhang et al. [14] recently found that the shear strengths of the joints are 3.3, 7.6, 14.9, 30.0 and $20.7 \mathrm{MPa}$ at the sintering temperatures of $180,200,220,250$ and $300^{\circ} \mathrm{C}$, respectively. Additionally, the fracture locations are at the interface with the substrate, within the sintered silver and at the interface with the chip when the joints are sintered at the temperatures of 180,250 and $300^{\circ} \mathrm{C}$. Thirdly, the internal micro-scale pores in the sintered silver paste also evolve during the thermal-mechanical service conditions. For example, the porosity of sintered silver layer keeps increasing during creep tests [15] and the silver grains of the sintered silver layer are found to be coarsened during high-temperature ageing [16]. By conducting ageing treatment at $250^{\circ} \mathrm{C}$ on tensile specimens, Choe et al. [17] observed the evaluation of microstructure of sintered hybrid $\mathrm{Ag}$ particles and found that the tensile strength deteriorates about $25 \%$ due to the coarsened microstructure in the first $200 \mathrm{~h}$ of ageing time and gradually recovers to the initial strength in the next $350 \mathrm{~h}$ of ageing time due to the reduction of porosity. From these three aspects, the porosity and microstructure are found to affect the mechanical properties of sintered silver pastes, however, the underlying mechanism for the corresponding correlation is rarely discussed in the existing studies especially from the microstructure point of view to emphasize the effect of proportion and distribution of silver particle size and shape.

In order to capture the critical factor of the proportion of particle sizes and shapes, two representative pressureless silver pastes consisting of particles at different size levels are adopted in this paper. That is, one silver paste is dominated by nanoparticles and the other is by particles or flakes at a micrometer scale. Thermal stability is firstly measured and followed up by nanoindentation experiments to evaluate the constitutive behaviour of these two sintered materials. Finally, the analytical constitutive models are discussed to correlate the sintered microstructure and the mechanical properties to facilitate and optimize the particle design of silver paste for the desired reliability of electronic packaging structures of high-power devices.

\section{Material description}

In this study, the silver paste dominated by nanoparticles was from Shenzhen Advanced Joining Technology Corp. and the silver paste dominated by particles and flakes at a micrometer scale was from KYOCERA Corp. Both of the silver pastes are formulated to be sintered without additional pressure. For the convenience of discussion, the former type of silver paste consisting of silver nanoparticles is denoted by 'AgNP', while the latter paste is denoted by 'AgMP' hereafter. The silver pastes before the sintering process were observed respectively using a field emission scanning electron microscope (SEM, JSM-7610F by JEOL) with a backscattered electrons imaging mode at the acceleration voltage of $15 \mathrm{kV}$.
The AgNP paste shown in Fig. 1(a) and (b) was found to be uniform and spherical and the particle diameter ranged up to $100 \mathrm{~nm}$ with the average diameter of about $26 \mathrm{~nm}$. As seen in Fig. 1(c) and (d), the AgMP paste was not so uniform and statistically measured by a particle size analyzer (Mastersizer 3000 by Malvern). Compared with Fig. 2(a) for the AgNP paste, a large portion of silver particles for the AgMP paste in Fig. 1(c) and (d) were found in Fig. 2(b) to be the flakes with the characterized size up to about $16.5 \mu \mathrm{m}$, while the average diameter of the involved submicron-sized Ag particles was $0.43 \mu \mathrm{m}$. It should be noted that no obvious aggregation or agglomeration was observed in both silver pastes.

\section{Methodology}

The thermal stability of silver pastes was characterized for the sintering process by the thermogravimetric analysis (TGA) using NETZSCH TG 209F3 and the differential scanning calorimetry (DSC) using TA Q20 under a pure Nitrogen flow rate of $20 \mathrm{ml} / \mathrm{min}$ and at a heating rate of $20^{\circ} \mathrm{C} / \mathrm{min}$ up to $450^{\circ} \mathrm{C}$. For DSC, the temperature is heated up to $450^{\circ} \mathrm{C}$ and then cooled down to the room temperature, which was followed up by a second heating and cooling process to confirm whether the exothermic peak from the desorption and vaporization of solvent or from the sintering and catalytic reactions in the paste [18]. In both TGA and DSC, the heat flow to the sample was measured as a function of time. The mass loss rate of silver pastes was analyzed by the derivative of thermogravimetric analysis (DTG) smoothed by the adjacent-averaging method in Origin 9.0.

In a pressureless manner, the sintering process was implemented at the temperature of $250^{\circ} \mathrm{C}$ for $1 \mathrm{~h}$ in the air on a hot plate with the ramping rate of about $10^{\circ} \mathrm{C} / \mathrm{min}$. In order to evaluate mechanical properties, nanoindentation experiments using a three-sided Berkovich diamond indenter in the Nano Indenter G200 by Agilent Technologies were conducted on the prepared disc-like samples. The prepared samples were mounted in polyvinyl chloride (PVC) tubes using the dental base acrylic resin powder. The solidified acrylic resin is capable of providing a matrix with a satisfactory hardness but no significant heat is released during solidification to affect the microstructure of sintered silver samples. By controlling the penetrating velocity of the Berkovich indenter, constant indentation strain rates can be achieved (i.e., $0.02 \mathrm{~s}^{-1}, 0.05 \mathrm{~s}^{-1}, 0.10 \mathrm{~s}^{-1}$ and $0.20 \mathrm{~s}^{-1}$ ) with the same maximum penetration depth of $2000 \mathrm{~nm}$. The substrate effect can be negligible, as the penetration is less than the $0.1 \%$ of the thickness. The nanoindentation results are analyzed based on the dimensionless approach for the Berkovich indenter with the selfsimilar characteristics. By utilizing the work done in the loading stage and the contact stiffness in the unloading stage, the material responses are correlated for nanoindentation and tension experiments. That is, the constitutive behaviour in the form of stress-strain relationship can be analytically extracted from the nanoindentation responses in the form of the indentation load-displacement response.

\section{Experimental result}

\subsection{Sintered morphology}

The sintered morphology was observed in Fig. 3. As driven by thermal energy input during the sintering process, a three-dimensional network-like porous structure in the sintered silver particles is ascribed to atom diffusion and neck formation between silver particles. Compared with the nanoparticles in Fig. 1(a), greater Ag grains were formed in Fig. 3(a) at the same magnification with the characteristic size ranging between $100 \mathrm{~nm}$ and $500 \mathrm{~nm}$ in the sintered AgNP. This means that dozens of AgNPs were connected by neck growths because of the Ag atoms diffusion during the sintering process. Due to uneven growth between adjacent particles, grain boundary gradually forms as a transition region in which some atoms are not exactly aligned. As a result, a greater $\mathrm{Ag}$ grain was formed by consuming smaller AgNP. Regarding AgMP 

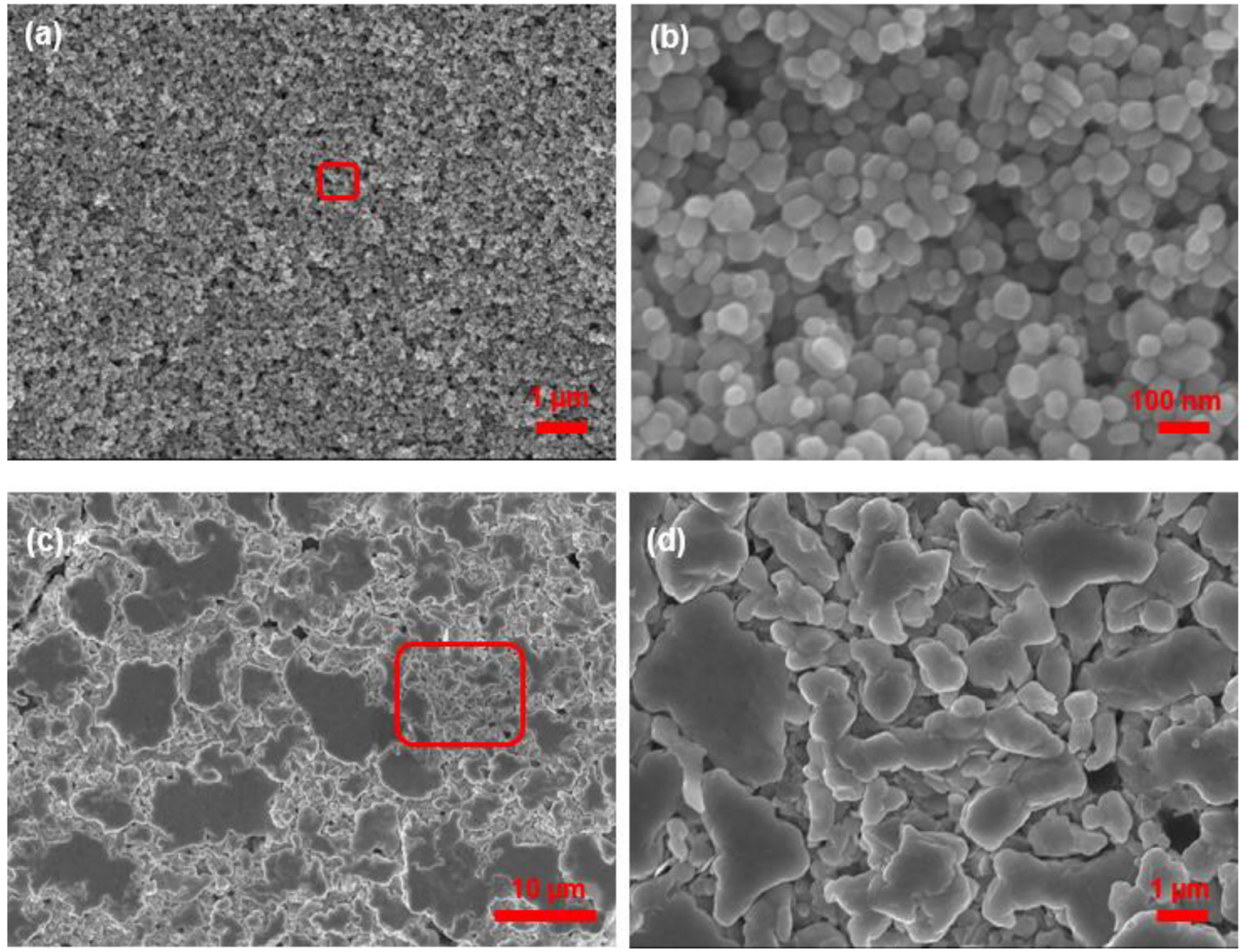

Fig. 1. Morphology of as-received silver pastes. (a) AgNP at the magnification of $10 \mathrm{k}$; (b) AgNP at the magnification of $100 \mathrm{k}$; (c) AgMP at the magnification of 2 $\mathrm{k}$; and (d) AgMP at the magnification of $10 \mathrm{k}$.
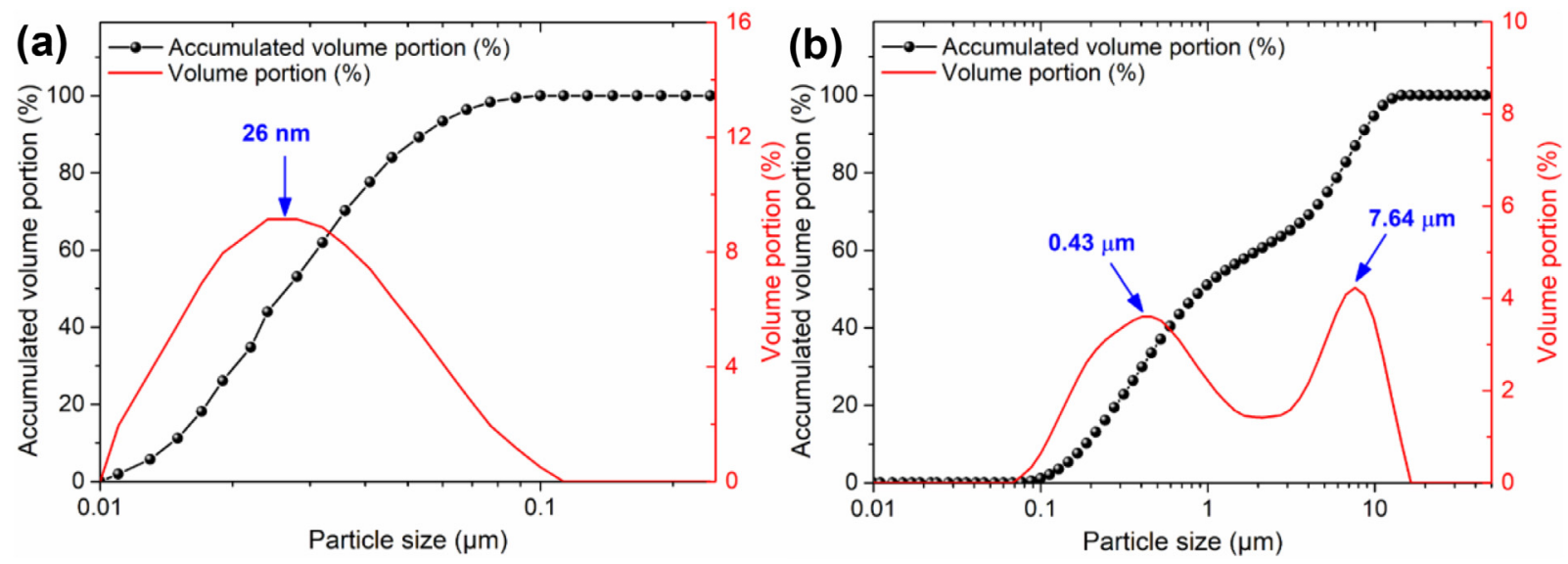

Fig. 2. Size distribution of the silver pastes. (a) AgNP; and (b) AgMP.

paste, the morphology change can be manifestly found in Fig. 1(d) and Fig. 3(b) before and after the sintering process. The submicron-sized Ag particles were connected to the adjacent flakes, which may result from the formation of sintering neck. The sintering neck results from the atom diffusion between particles so that a network is formed after the sintering process under the provided temperature profile. Nevertheless, the size of silver flakes did not dramatically increase. It can be concluded that compared with microparticles, the nanoparticles are more prone to diffuse and form necks between smaller particles. Thus, it is speculated that the atom diffusion is more active on the smaller sized spherical particles with higher surface energy, which will form the sintering necks and also efficiently increase the sizes of Ag particles. On the other hand, the lower surface energy on the silver flakes impedes the atom diffu- sion despite the thermal energy input during the sintering process. This elucidates the intrinsic difference of the sintering mechanism between these two types of silver pastes. Apparently, the microstructure of sintered AgMP was not as uniform as that of sintered AgNP, which leads to the distinguishing mechanical, electrical and thermal properties [19].

\subsection{Thermal stability}

For AgNP paste, Fig. 4(a) shows that the mass loss onsets at $59.8^{\circ} \mathrm{C}$ and ends at $142.6{ }^{\circ} \mathrm{C}$ with an accumulated mass change of $12 \%$. The mass loss can be reflected by the mild exothermic peak between $81.2^{\circ} \mathrm{C}$ and $143.2^{\circ} \mathrm{C}$ in the 1 st run of DSC heating results in Fig. 4(b). As an exothermic peak is not evident in the 2nd run of DSC heating, the mass 

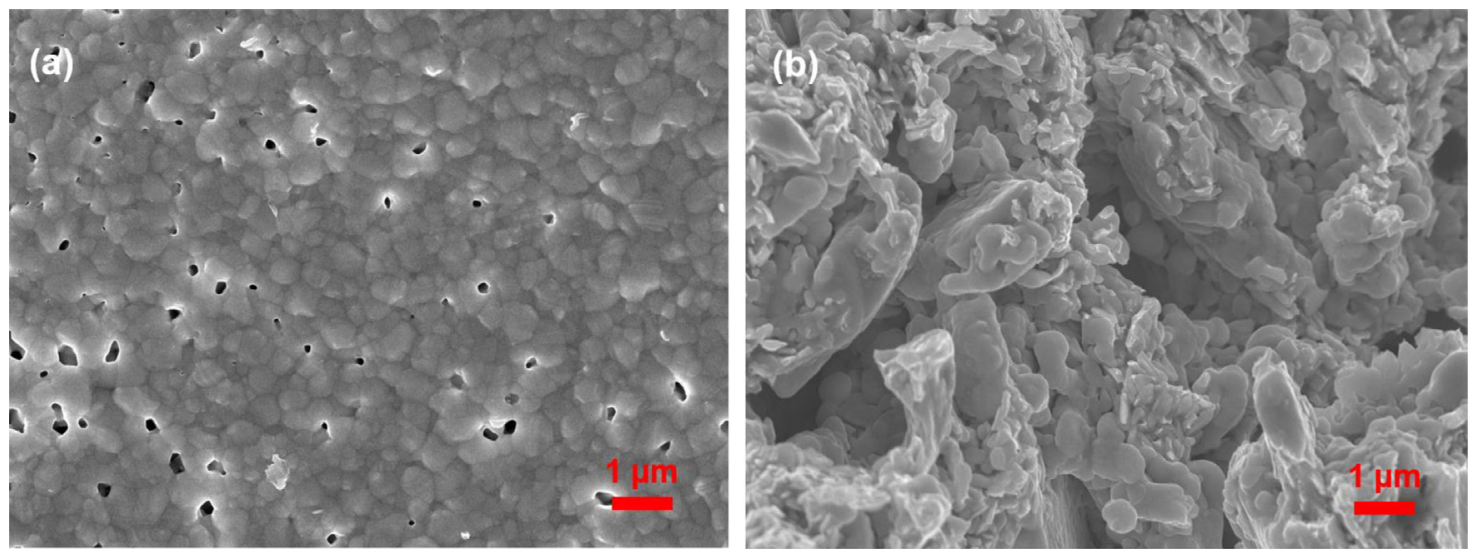

Fig. 3. Morphology of sintered silver pastes at the magnification of $10 \mathrm{k}$. (a) AgNP; and (b) AgMP.
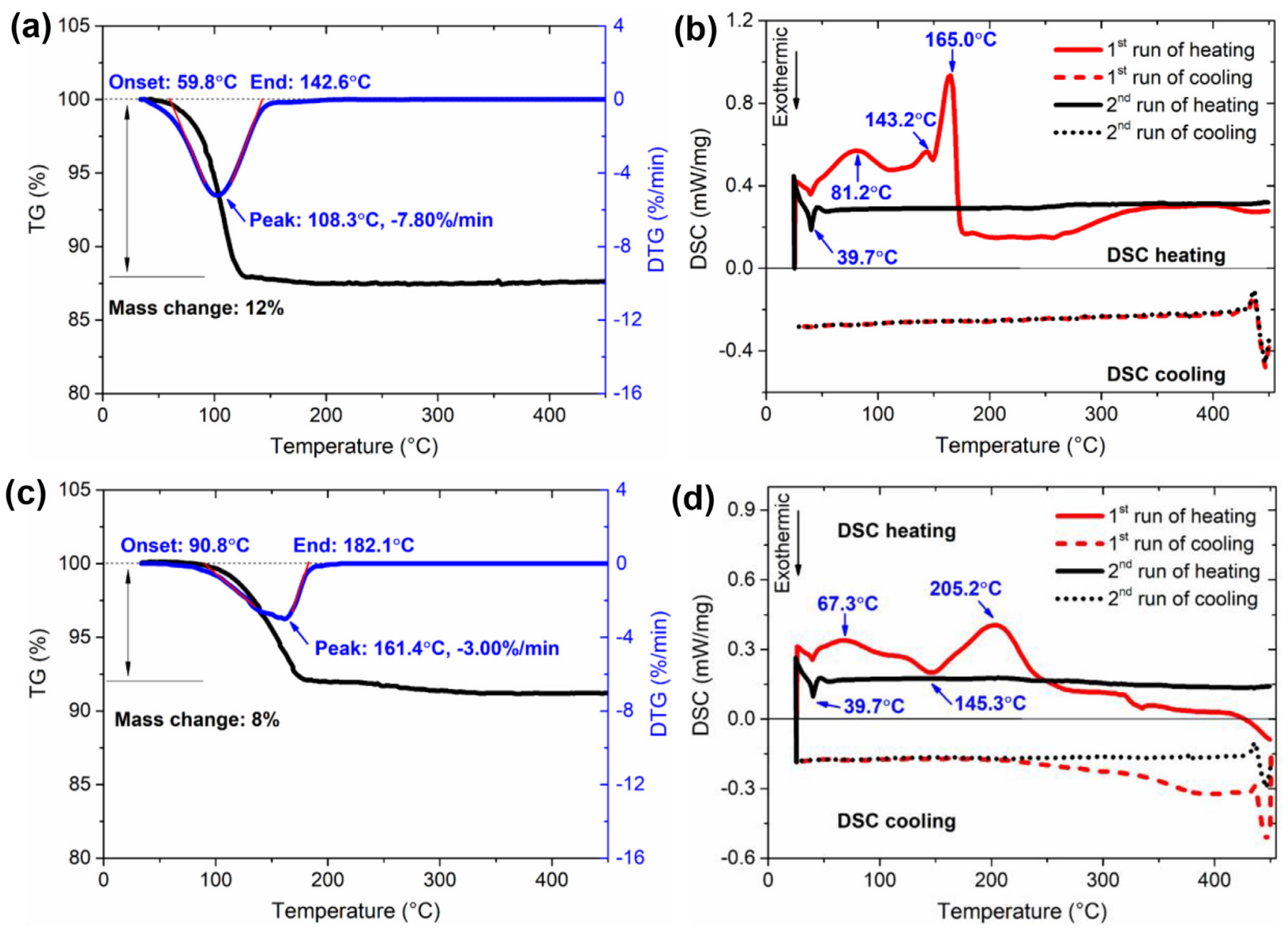

Fig. 4. Thermal stability of silver pastes. (a) TG and DTG results of AgNP; (b) DSC result of AgNP; (c) TG and DTG results of AgMP; and (d) DSC result of AgMP.

loss resulted from desorption and vaporization of solvent. The sharp peak at $165.0^{\circ} \mathrm{C}$ is speculated to be induced from the intensive sintering process and catalytic reactions between binder and silver particles. For AgMP paste, the accumulated mass change is $8 \%$ between the temperatures of $90.8^{\circ} \mathrm{C}$ and $182.1{ }^{\circ} \mathrm{C}$ in Fig. 4(c), which occurred at the mild exothermic peak around $145.3^{\circ} \mathrm{C}$ as shown in Fig. 4(d).

Compared with AgNP paste, the mass loss is less but the temperature for desorption and vaporization of solvent is higher. This may result from the more efficient sintering process for the silver pastes with smaller spherical sized particles which occupy higher surface energy compared with the flakes in the AgMP. Additionally, the sintering temperature of AgMP is indicated by the sharp endothermic peak at $205.2^{\circ} \mathrm{C}$. This means a greater size of silver particles with micro-sized flakes requires a higher temperature for the sintering process. But the mass loss for the silver paste with smaller particles during the sintering process is greater as the mass ratio of vaporizable binder is higher to avoid aggregation or agglomeration of nanoparticles. Thus, the silver paste with smaller sized spherical particles can be efficiently sintered to achieve the thermally stabilized state with lower thermal energy input during the sintering process such as lower sintering temperature and shorter sintering duration. This thermally stabilized state is speculated to contribute the compact microstructure with minimized pores 

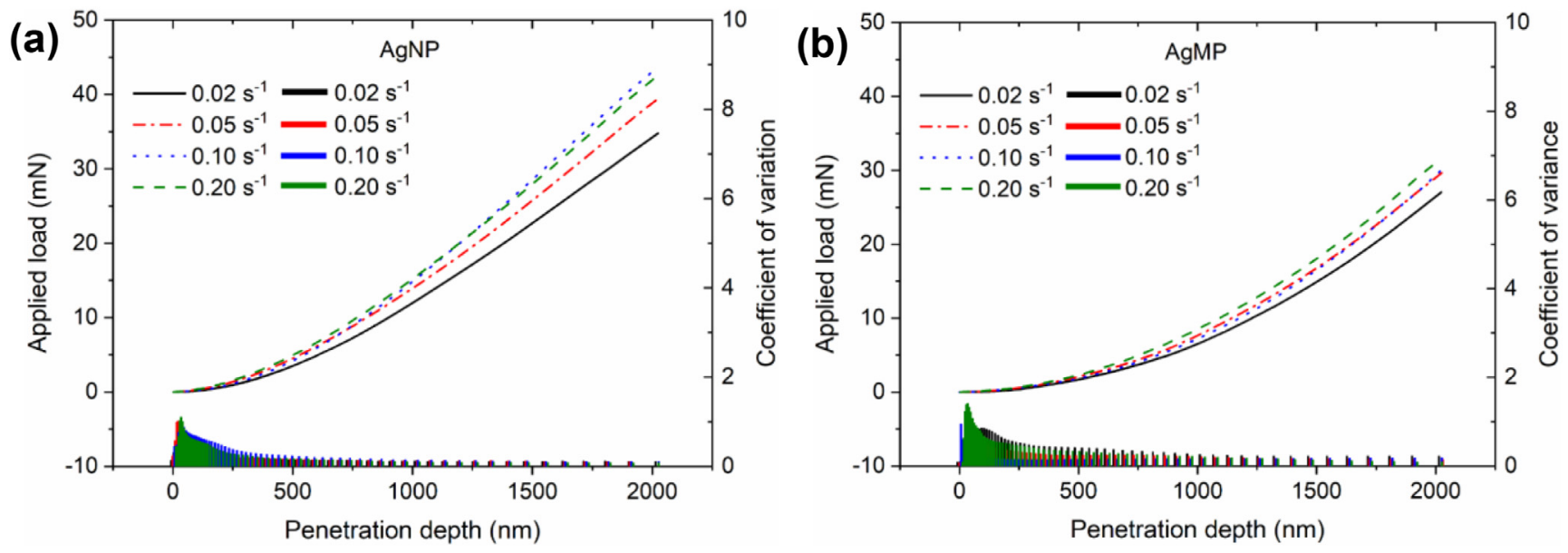

Fig. 5. Nanoindentation responses of sintered samples. (a) AgNP; and (b) AgMP.

as shown in Fig. 3(b) and thus benefit the mechanical properties of sintered AgNP by avoiding the damage initiation from fewer micro-defects such as voids and cracks.

\subsection{Nanoindentation response}

The measured nanoindentation responses are illustrated in the form of indentation load-displacement relationship in Fig. 5. In order to evaluate the result deviation among the repeated nanoindentation experiments in the same condition, the coefficients of variation (COVs) are incorporated as the function of penetration depth by the bar chart. According to Fig. 5, both samples show an increasing applied load for a greater penetration depth. Meanwhile, the strain rate effect is also observed especially at the lower indentation strain rates of $0.02 \mathrm{~s}^{-1}$ and $0.05 \mathrm{~s}^{-1}$. At the initial penetration, the COVs are relatively high due to the porous microstructure and roughness on the sample surfaces. Subsequently, the COVs are approaching stabilized values when the penetration depth is greater than $1500 \mathrm{~nm}$, which can be accepted as the location to reliably attain the mechanical properties such as Young's modulus and hardness. At the same penetration depth, the applied load on AgNP is obviously greater than that on AgMP. This indicates the mechanical strength is higher for AgNP due to the smaller size of $\mathrm{Ag}$ particles.

The fundamental mechanical properties such as Young's modulus and hardness can be measured as the functions of penetration depth during the loading stage by taking advantage of the contact stiffness measurement (CSM) technique [20]. Compared with the CSM technique, the classical Oliver-Pharr (OP) method proposed by Oliver and Pharr [21] strongly relies on the contact stiffness measured from the initial slope of nanoindentation responses. Nevertheless, the determination of contact stiffness is challenging to objectively obtain. The initial slope of nanoindentation responses varies so quickly that different ranges of the unloading curve numerically fitted by using a power law function will lead to different values of contact stiffness. For instance, the range between $40 \%$ and $98 \%$ of the maximum applied load is suggested as the default value for the Nanoindentation tester $\mathrm{NHT}^{2}$ produced by Anton Parr. Another challenge for the OP method is that the true contact area is difficult to be accurately measured due to the pile-up and sink-in deformation around the indenter during the indentation process. Therefore, the values of Young's modulus are greatly underestimated by the OP model as found by Martin and Troyon [22]. In this study, the CSM technique is adopted to calculate in the manner proposed by Hay et al. [20] by considering the frame stiffness and the real part of the amplitude ratio when evaluating the Young's modulus. So the CSM approach reflects the influence of the amplitude of the applied oscillating force, the amplitude of the displacement response and also the phase shift.

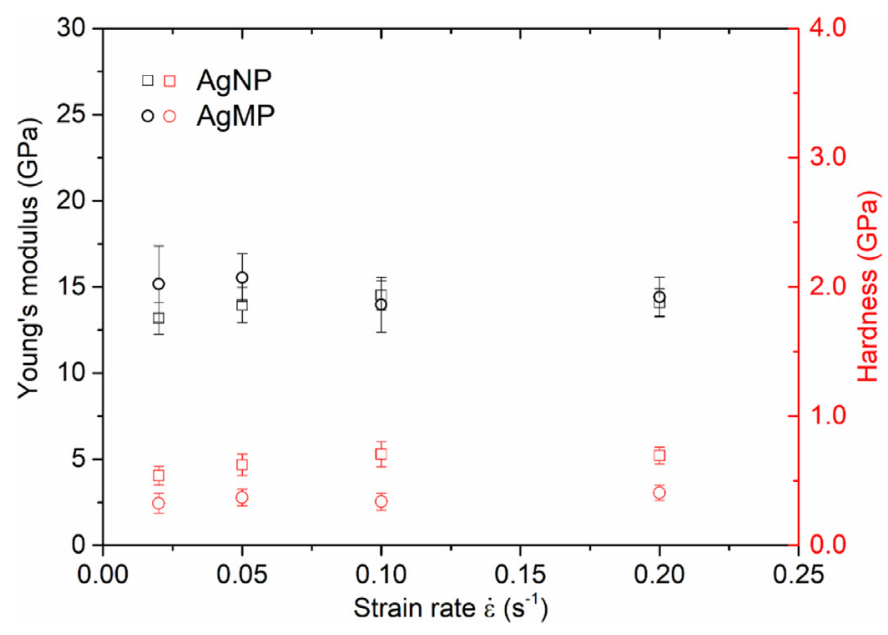

Fig. 6. Young's modulus and hardness of sintered silver materials.

In Fig. 6, the stabilized values of Young's modulus and hardness are achieved at the penetration depth between $1500 \mathrm{~nm}$ and $1600 \mathrm{~nm}$, which is believed to be an appropriate range of penetration depth to reliably measure the mechanical properties of sintered sample according to Fig. 5. These values are at similar levels with the recently reported values by indentations with multiple strain-rate jumps [7]. The comparison in Fig. 6 indicates that no significant difference in the aspect of Young's modulus results from the microstructure between AgNP and AgMP. However, the hardness of sintered AgNP is slightly greater than that of AgMP, which means the yield strength of AgNP is expected to be greater due to the linear association between hardness and yield strength as found by Tabor [23] and Carsley et al. [24]. This might be deemed as a criterion to correlate the microstructure and the mechanical behaviour using nanoindentation approach, despite that the hardness is not incorporated as a parameter in the calculation of the proposed constitutive model.

\section{Constitutive model}

There are a few numerical and analytical approaches available to extract the constitutive behaviour in the form of stress-strain relationship from the nanoindentation responses subjected to different indenters such as conical and spherical indenters [7,25-30]. By overcoming the difficulty of solution uniqueness, the analytical approach proposed by Ogasawara et al. [31] and recently improved by Long et al. [32] is utilized to measure the plastic properties of a power-law constitutive model 
in Eq. (1) for tensile behaviour under the uniaxial loading in the present study.

$\sigma=R \varepsilon^{n}$

where $\sigma$ is the stress, $\varepsilon$ is the strain, $n$ is the hardening exponent, and $R$ is the work-hardening rate. As for the representative stress $\sigma_{R}$, the corresponding $\varepsilon_{R}$ is the representative strain which is defined as the plastic strain during the axisymmetric deformation. By following the relationship in Eq. (1), the representative stress $\sigma_{R}$ and the corresponding $\varepsilon_{R}$ can be correlated in Eq. (2).

$\sigma_{R}=R\left(\varepsilon_{R}\right)^{n}$

As deduced by Alkorta et al. [33], the applied strain rate, $\dot{P} / P$, is closely related to hardness and the penetration depth as presented in Eq. (3). As the hardness is not significantly time-dependent, the term $\dot{H} / H$ can be assumed to be zero. In Eq. (3), the ratio of the instantaneous change in the applied load with the instantaneous applied load, $\dot{P} / P$, is approximately equal to two times the ratio of the instantaneous change in the penetration depth with the instantaneous penetration depth $h / h$. Apparently, the ratio $\dot{h} / h$ is of the dimension of strain rate $\dot{\varepsilon}$ and thus is defined and widely used as the indentation strain rate for nanoindentations [20]. For convenience, the term 'strain rate' is used for the indentation strain rate afterwards unless stated otherwise.

$\frac{\dot{P}}{P}=\frac{\dot{H}}{H}+2 \frac{\dot{h}}{h} \approx 2 \frac{\dot{h}}{h}$

where $P$ is the applied indentation load, $H$ is the hardness, and $h$ is the penetration depth. The dot notation represents the rate of corresponding variable.

Essentially, based on the self-similarity of a Berkovich indenter, the work done in the loading stage and the contact stiffness in the unloading stage are taken into account to assess the representative stress $\sigma_{R}$ and the hardening exponent $n$, respectively. To correlate the material responses during nanoindentation and tension experiments, the representative stress $\sigma_{R}$ and the hardening exponent of $n$ are dependently enriched by the rate factors $\psi_{\sigma}(=0.56)$ and $\psi_{n}(=0.33)$ as calibrated by Long et al. [32]. Regarding the loading stage with the increasing applied load, the first dimensionless analysis based on numerical fitting is written in Eq. (4).

$\frac{W_{t}}{\delta_{\max }^{3} \times \sigma_{R} / \psi_{\sigma}}=-0.20821 \xi^{3}+2.6502 \xi^{2}-3.7040 \xi+2.7725$

where $W_{t}$ is the indentation work done determined by the area integration in the loading part until the maximum penetration depth $\delta_{\max }$, and $\sigma_{R}$ is the representative stress corresponding to the representative strain $\varepsilon_{R}$ which is determined from the numerical fitting $\varepsilon_{R}=0.0319 \cot (\alpha)$ for a Berkovich indenter with the half angle of $65.27^{\circ}$ [34].

The dimensionless variable $\xi=\ln \left(\bar{E} / \sigma_{R} \cdot \psi_{\sigma}\right)$ is related to the plain strain modulus $\bar{E}=E /\left(1+v^{2}\right)$ where $E$ is the Young's modulus which is obtained from the CSM technique as shown in Fig. 6 and $v$ is the Poisson's ratio which is approximated to be 0.37 by ignoring the porosity effect $[9,35]$. Thereafter, the second dimensionless analysis regarding the initial unloading stage is written in Eq. (5) with the function of $\vartheta=n / \psi_{n}$ for fitting the normalized contact stiffness $S$. The dimensionless analysis is based on extensive finite element simulation results by Ogasawara et al. [31]. The values from the left and right dimensionless terms in Eqs. (4) and (5) do reproduce the characteristic loading and unloading responses of nanoindentation deformations. In the aspect of mechanical behaviour, it is found that the work done in the loading stage determines the representative stress while the hardening exponent is dominated by the contact stiffness.

$\frac{S}{2 \delta_{\max } \bar{E}}=A \xi^{3}+B \xi^{2}+C \xi+D$

where $A=-0.04783 \vartheta^{2}+0.04667 \vartheta-0.01906, B=0.6455 \vartheta^{2}-0.6325 \vartheta$ $+0.2239, C=2.2989^{2}+2.0259-0.4512$, and $D=2.050 \vartheta^{2}-1.502 \vartheta$ +2.109 .

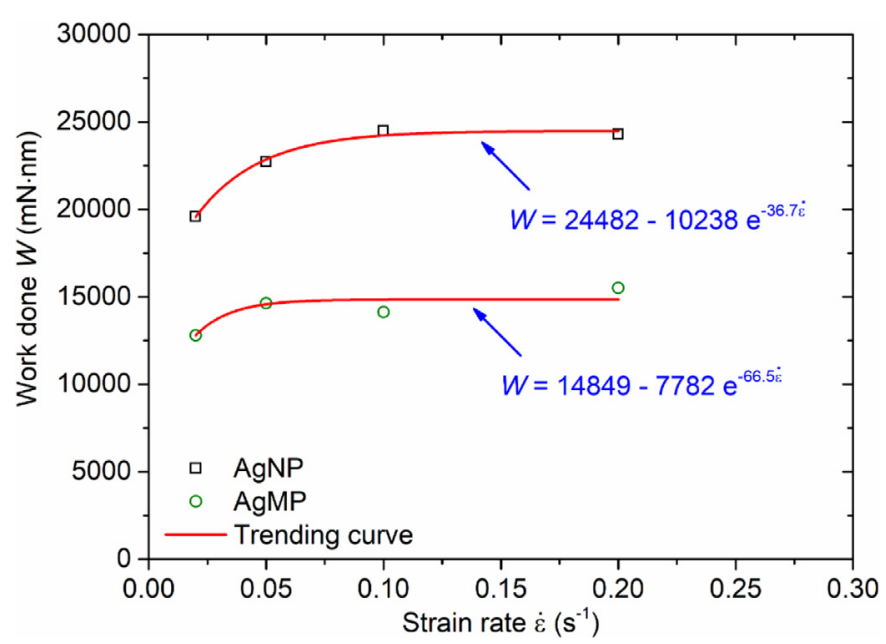

Fig. 7. Work done during the nanoindentation loading stage on sintered materials.

As the load resistance on AgNP at the same penetration depth is significantly greater than that on AgMP in Fig. 5, therefore, the integrated work done on AgNP during the loading stage is definitely greater. This coincides with the finding as observed in Fig. 7. It should be noted that the COVs in the initial penetration as shown in Fig. 5 are higher because of the surface roughness of porous microstructure. As the penetration depth increases, the porous microstructure is more densified with sufficient contact between indenter and the surrounding material. Therefore, more averaged properties can be extracted with smaller COVs. Consequently, the integration of work done in this study takes into account the contribution of the indentation process after the initial penetration, which is about $70 \%$ of the whole area compassed by the loading stage. It is also found in Fig. 7 that the work done seems to approach the intrinsic limits of $24482 \mathrm{mN} \bullet \mathrm{nm}$ and $14849 \mathrm{mN} \bullet \mathrm{nm}$ at higher indentation strain rates for AgNP and AgMP, respectively, which can be estimated based on the fitting curves by exponential functions with negative exponents. Furthermore, the AgNP is more sensitive to the indentation strain rate compared with AgMP. However, both sintered materials become insensitive to the indentation strain rate greater than $0.10 \mathrm{~s}^{-1}$. The saturated work done offers the opportunity to optimize the mechanical properties of sintered AgNP and AgMP materials for high strain rate applications.

As both the left and right terms of Eq. (4) are the functions of the representative stress $\sigma_{R}$, the left and right terms of Eq. (4) can be numerically obtained by varying the $\sigma_{R}$ value as shown in Fig. 8 with the rate factor $\psi_{\sigma}(=0.56)$ for the representative stress $\sigma_{R}$. Therefore, by utilizing the work done in Fig. 7, the representative stress $\sigma_{R}$ can be numerically solved at the intersection of the two curves from the parameterized left and right terms in Eq. (4) as shown in Fig. 8. Apparently, the right term does not vary dramatically while the work done in the left term dominates the solution of representative stress.

After solving the representative stress $\sigma_{R}$, the hardening exponent $n$ is left to be the only unknown in Eq. (5), as the contact stiffness $S$ can be measured from the initial slope of unloading stage of indentation load-displacement responses. For instance, the contact stiffness for the nanoindentations at the indentation strain rate of $0.02 \mathrm{~s}^{-1}$ is measured for both AgNP and AgMP samples in Fig. 9. Thus, the averaged contact stiffness with different indentation strain rates in Fig. 10 can be employed to similarly solve Eq. (5) as demonstrated in Fig. 11.

Compared with AgNP in Fig. 10(a), the contact stiffness of AgMP in Fig. 10(b) apparently shows more random distributions and the averaged value almost remains a constant value. Nevertheless, the contact stiffness of AgNP increases with the indentation strain rate and approaches to be $148.7 \mathrm{kN} / \mathrm{m}$ between $0.10 \mathrm{~s}^{-1}$ and $0.20 \mathrm{~s}^{-1}$, which can be well fitted by an exponentially based function with a negative exponent. Compared with the constant contact stiffness with a greater 


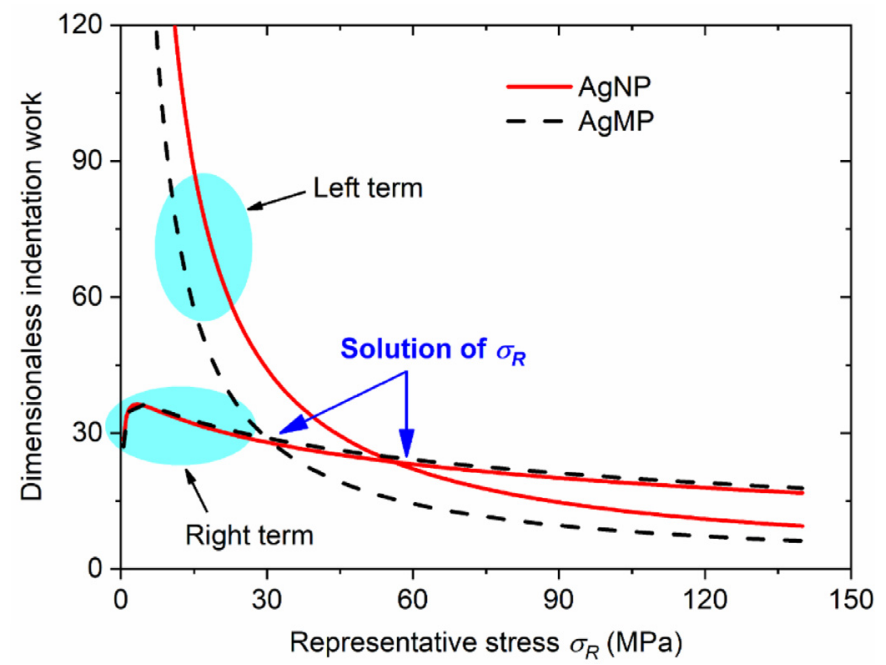

Fig. 8. Numerical solution of Eq. (4) for the nanoindentation at the indentation strain rates of $0.02 \mathrm{~s}^{-1}$.

deviation of sintered micro-size particles or flakes, the sintered nanoparticles in the silver paste form the microstructure with a smaller contact stiffness which is more strain-rate dependent and regularly distributed to approach a stabilized value above the indentation strain rate of 0.10 $\mathrm{s}^{-1}$.

Similar to Fig. 8, the right term of Eq. (5) is the function of the hardening exponent $n$ with the left term as a known quantity from the nanoindentation response, so the right term of Eq. (5) can be numerically obtained by varying the $n$ value as shown in Fig. 11 . The left term of Eq. (5) is the ratio between $S$ and $2 \delta_{\max } \bar{E}$, thus the y-axis of Fig. 11 is referred as the dimensionless contact stiffness. As the red curves are lower than the black dash curves in Fig. 11, the microstructure of sintered AgNP sample leads to smaller values for both left and right terms in Eq. (5). Since the intersection is achieved with a greater value of hardening exponent, it can be deduced that the solution of Eq. (5) is dominated by the contact stiffness in the left term rather than the hardening exponent in the right term.

It should be noted that the material responses under nanoindentation so far are measured in terms of the applied load-displacement curves. Due to the continuous plastic deformation under the indenter as the indentation depth increases, the spherical indenter is believed to be more appropriate for extracting the average properties of porous materials, while the Berkovich indenter provides more localized properties [30]. Besides, the plastic deformation under the pyramidal or conical indenters remains constant due to the self-similar geometry [36]. Thus, the localized responses extracted by the Berkovich indenter can be approximately adopted as the intrinsic material property for further evaluating the constitutive model in terms of stress-strain response. Compared with the bulk material under uniaxial tests, the stress-strain response from nanoindentations has to be related to the constitutive response using the proposed rate factors to overcome the gap between the respective strain rates of the uniaxial and nanoindentation tests. As observed from the calibration for the lead-free solder materials, the stress-strain responses can be phenomenologically equivalent between uniaxial and nanoindentation tests given that uniaxial strain rate and indentation strain rate can be reasonable correlated, which therefore can be deemed to be a generalized approach. Nevertheless, it should be pointed out that the sintered porous structure may have a certain effect on the rate factors, which is urgently required to find out a suitable combination of sintered porous silver material and testing machines to validate or recalibrate the rate factors using both uniaxial and indentation tests.

By performing numerical solutions similar to Figs. 10 and 11, the values of the representative stress $\sigma_{R}$ and the hardening exponent $n$ for all the indentation strain rates of both sintered samples can be obtained with the rate factors $\psi_{\sigma}$ of 0.56 and $\psi_{n}$ of 0.33 according to the recently published work by the authors [32]. It is reasonably assumed [28] that the elastic behaviour of sintered materials obey the relationship of $\sigma_{e}=E \varepsilon_{e}$ until the occurrence of plasticity when the calculated elastic stress is less than the plastic stress from Eq. (1). This transition from elastic to plastic stress is deemed as the yielding occurrence, where the yield strength is determined. As the representative stress $\sigma_{R}$ corresponds to the representative strain $\varepsilon_{R}$ as explained about Eq. (4), the work-hardening rate $R$ can be calculated according to Eq. (2) with the previously determined hardening exponent $n$. Therefore, the power-law constitutive model is completed as plotted in Fig. 12 and the associated parameters in Eq. (1) are listed in Table 1. The stressstrain relationship of sintered materials at different uniaxial strain rates are calculated until the uniaxial strain of 0.1 which is believed to be less than the ultimate strain of the ductile pure silver but a sufficient strain for material deformation before failure for the finite element simulations of electronic packaging structures.

In Fig. 12, it is observed that both yield strength and hardening behaviour of sintered AgNP are much greater than that of AgMP due to the increase of work done in the loading stage and the decrease of contact stiffness in the unloading stage during the nanoindentation experiments.
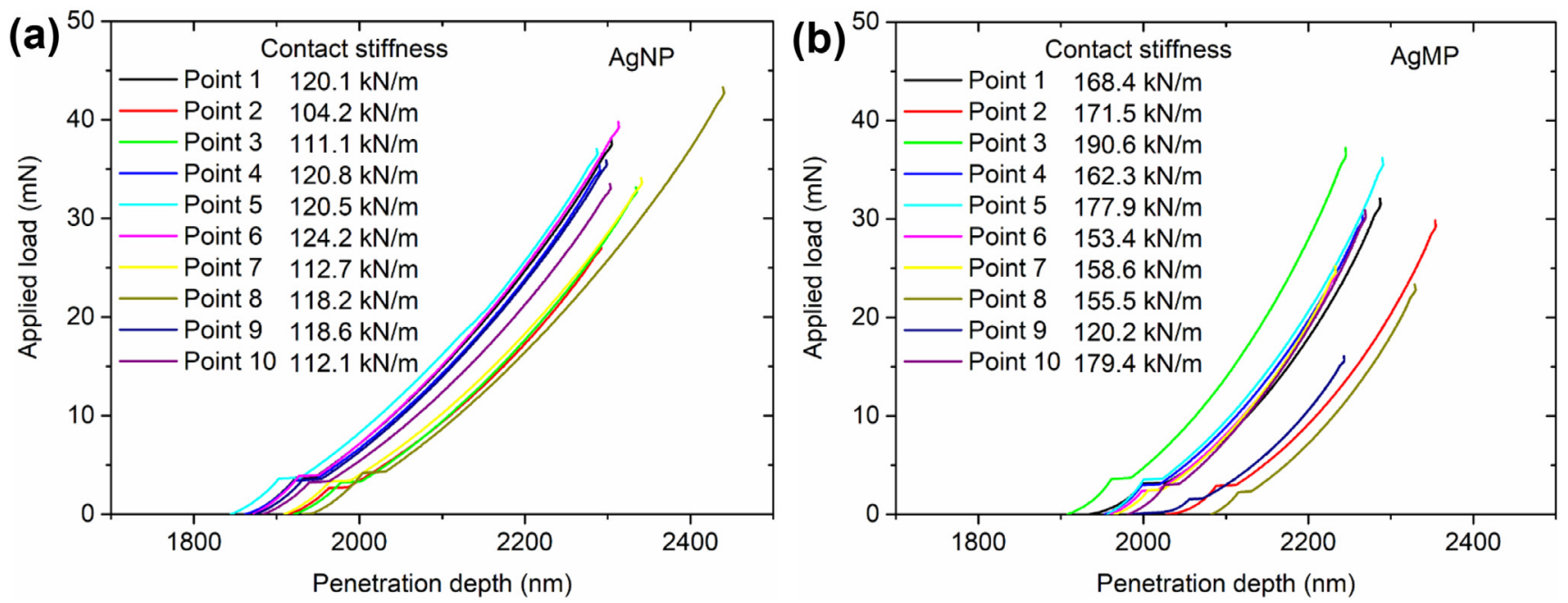

Fig. 9. Contact stiffness measured by nanoindentation at the indentation strain rate of $0.02 \mathrm{~s}^{-1}$. (a) AgNP; and (b) AgMP. 

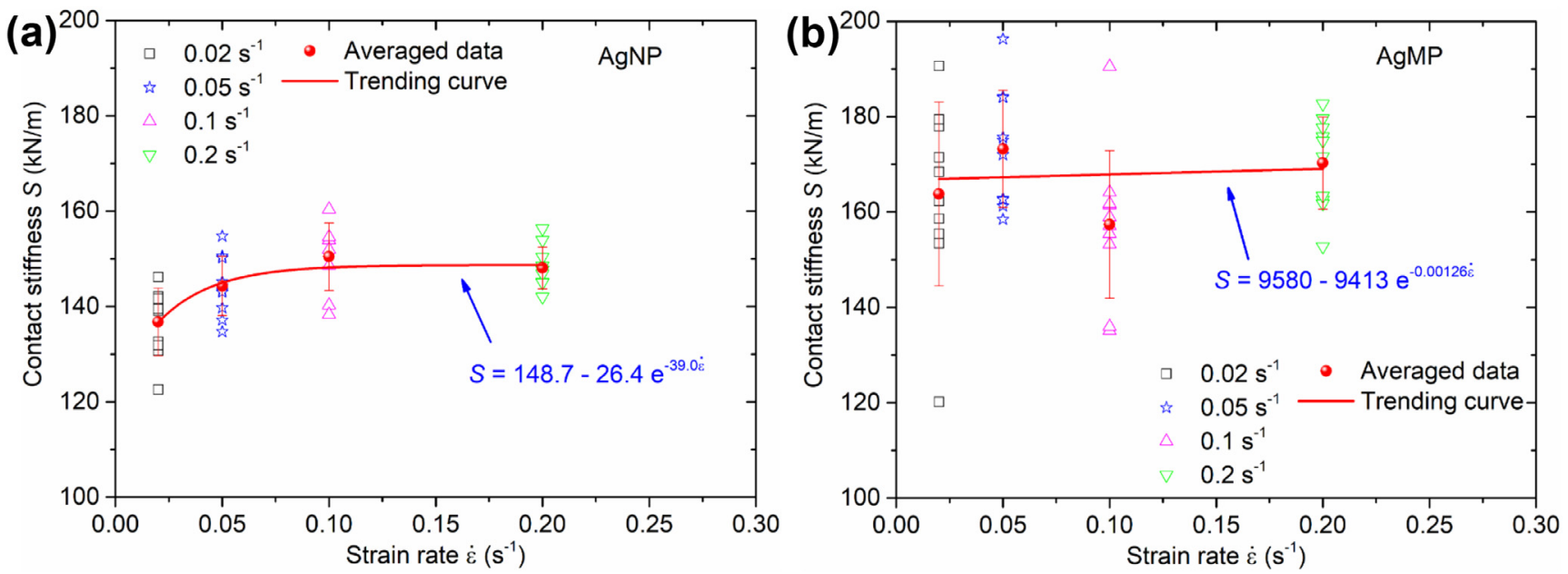

Fig. 10. Contact stiffness of sintered materials subjected to different indentation strain rates. (a) AgNP; and (b) AgMP.

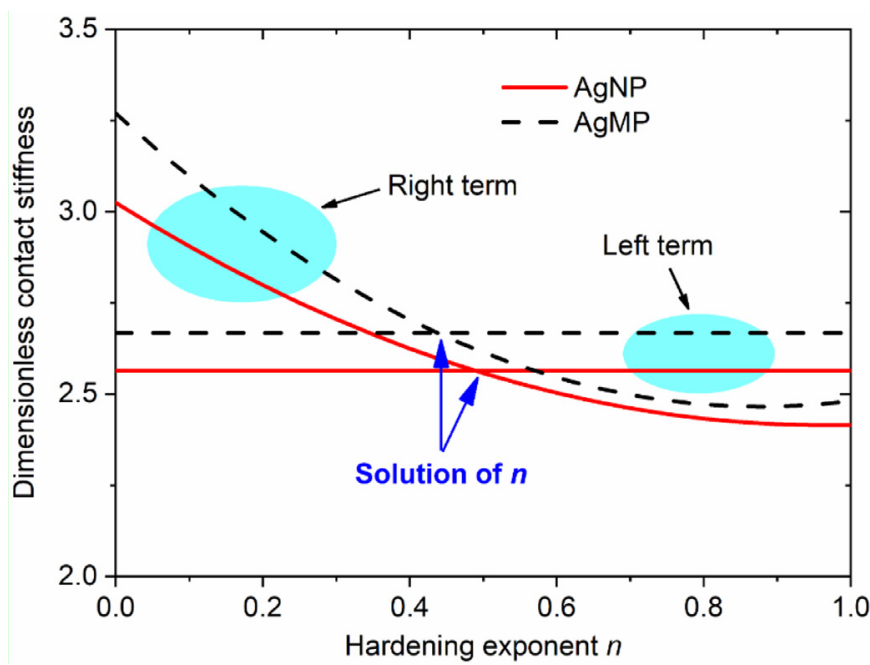

Fig. 11. Numerical solution of Eq. (5) for the nanoindentation at the indentation strain rate of $0.02 \mathrm{~s}^{-1}$.

This agrees well with the discussion about Fig. 6 based on the fact that the hardness of sintered AgNP is slightly greater than that of AgMP. Due to the densification of the porous microstructure, the measured strength of AgNP is approaching the level of the silver ultimate strength [37]. In addition, Table 1 shows that compared with AgNP, the hardening exponent of AgMP decreases significantly with the increasing indentation strain rate. This means the effect of particle size in the silver paste determines the constitutive behaviour of sintered materials. In order to achieve the desirable electronic packaging structures in the tradeoff between manufacturing cost and product quality, the findings in this paper offer the opportunity to optimize the mechanical reliability by tuning

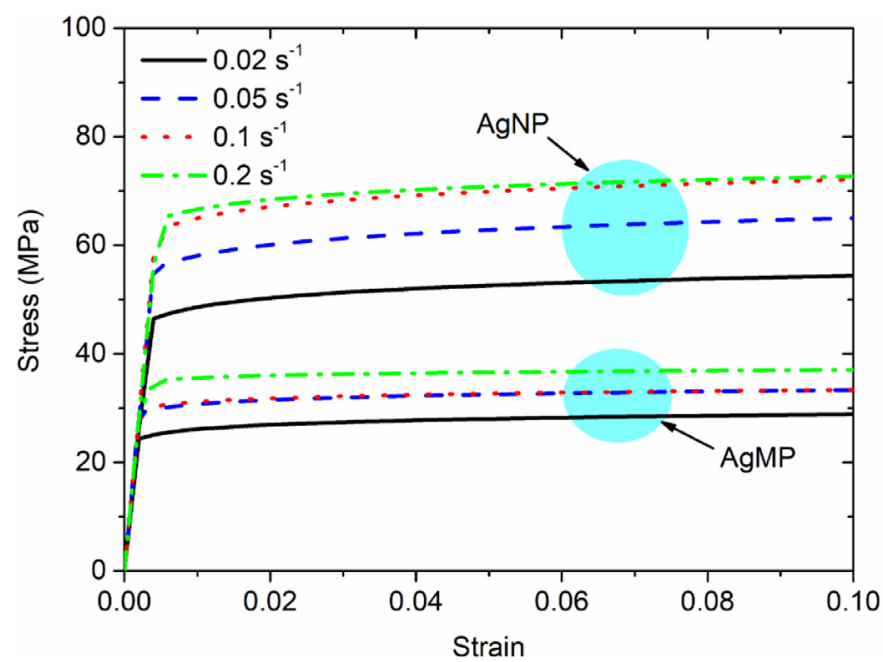

Fig. 12. Stress-strain relationship of sintered materials at different uniaxial strain rates.

the particle size and shape in the silver paste as one of the promising die-attach materials for high-temperature electronic applications

\section{Conclusion and discussion}

By comparison of two sintered silver pastes with different characteristic sizes and shapes, the thermal stability, mechanical properties (such as Young's modulus and hardness) and the parameters of the power-law constitutive model are measured and analyzed. Compared with microscale particles and flakes in the AgMP paste, silver nanoparticles in the AgNP paste requires a lower temperature for sintering process but also

Table 1.

Parameters of the constitutive model for sintered silver materials.

\begin{tabular}{lllllll}
\hline Material & Uniaxial strain rate $\left(\mathrm{s}^{-1}\right)$ & $E(\mathrm{GPa})$ & $\sigma_{R}(\mathrm{MPa})$ & $S(\mathrm{kN} / \mathrm{m})$ & $n$ & $R(\mathrm{MPa})$ \\
\hline AgNP & 0.02 & 13.2 & 52.0 & 136.8 & 0.049 & 60.9 \\
& 0.05 & 13.9 & 62.1 & 144.3 & 0.049 & 72.7 \\
& 0.10 & 14.5 & 69.2 & 150.4 & 0.045 & 80.0 \\
\multirow{4}{*}{ AgMP } & 0.20 & 14.1 & 70.2 & 148.1 & 0.038 & 79.2 \\
& 0.02 & 15.2 & 27.7 & 163.8 & 0.044 & 31.9 \\
& 0.05 & 15.5 & 32.3 & 173.2 & 0.035 & 36.1 \\
& 0.10 & 14.0 & 32.5 & 157.4 & 0.030 & 35.7 \\
\hline
\end{tabular}


shows a greater mass loss because of higher desorption and vaporization of solvent. The correlation between microstructure and constitutive behaviour is analytically revealed based on the experimental results from nanoindentations. Compared with AgMP, the sintered AgNP results in a slight increase of hardness but no effect is observed for Young's modulus. After solving the nonlinear equations from dimensionless analysis, the power-law based constitutive model can be obtained.

The work done approaches the intrinsic limits at higher indentation strain rates for AgNP and AgMP. Compared with AgMP, the contact stiffness of the AgNP material is less with a smaller deviation, but it is more strain-rate sensitive and stabilizes only when the indentation strain rate is greater than $0.10 \mathrm{~s}^{-1}$. More importantly, by scaling down the characteristic size of silver particles from micrometer to nanometer, both yield strength and hardening behaviour of sintered materials are much greater. In light of the effect of particle size and shape on the mechanical behaviour of sintered materials, it is feasible to optimize the mechanical reliability by tuning the particle size and shape in the silver paste to be utilized as the die-attach material for high temperature applications of electric packaging structures. Nevertheless, more fundamental studies should be carried out to further investigate the effects of particle size and shape from the surface energy point of view.

It is noteworthy that the proposed constitutive model in this paper is more applicable to compressive behaviour of sintered silver materials. However, the ultimate strain when the sintered material fails upon the deformation capacity at extremely large deformation is not available in the proposed analytical approach. The model should be utilized cautiously to describe the tensile and shear behaviour, since these failures are prone to be induced by micro-scale defects in the test samples and no obvious plastic behaviour is usually observed. Therefore, the plastic behavior in the proposed model may be pronounced as experimentally measured; however, the measured Young's modulus and hardness still stand. Furthermore, the proposed model is solely for describing the constitutive behaviour of sintered silver materials, therefore it cannot be adopted to predict the interfacial bonding failure due to the mismatch of thermal expansion between different materials in the electronic packaging structures.

It is usually believed that the finer microstructure with smaller size of $\mathrm{Ag}$ grains benefits the mechanical strength of sintered $\mathrm{Ag}$ particles, which can be confirmed by the fact that ductile deformation is distinct and the fracture mainly happened at the sintered silver layer [14]. However, there is a certain competition between grain coarsening and porosity reduction not only during the sintering process but also in the service life at high temperatures. As demonstrated by examining the microstructure evolution during the sintering process in an independent study to be published by the authors, grain coarsening will further consume the smaller Ag particles and reduce the pore number, but increase the porous volume at the same time. Therefore, whether the grain coarsening dominates the high temperature service life can be determined by examining the deterioration of mechanical properties of sintered $\mathrm{Ag}$ particles in future studies.

\section{Acknowledgments}

This work was supported by the National Natural Science Foundation of China (No. 51508464), the Natural Science Foundation of Shaanxi Province (No. 2017JM1013), the Astronautics Supporting Technology Foundation of China(No. 2019-HT-XG), and the Fundamental Research Funds for the Central Universities (No. 3102018ZY015). C.C. acknowledges the PhD Research Startup Foundation of Taiyuan University of Science and Technology(No. 201704).

\section{References}

[1] Yu DJ, Chen X, Chen G, Lu GQ, Wang ZQ. Applying Anand model to low-temperature sintered nanoscale silver paste chip attachment. Mater Design 2009;30:4574-9.
[2] Morkoç H, Strite S, Gao GB, Lin ME, Sverdlov B, Burns M. Large-band-gap SiC, III-V nitride, and II-VI ZnSe-based semiconductor device technologies. J Appl Phys 1994;76:1363-98.

[3] Strite S, GaN Morkoc H. AlN and InN: a review. J Vac Sci Technol B 1992;10:1237-66.

[4] Lu GQ, Calata JN, Zhang Z, Bai JG. High density microsystem design and packaging and component failure analysis. In: HDP '04 Proceeding of the Sixth IEEE CPMT Conference on, 2004; 2004. p. 42-6.

[5] Chen G, Zhang ZS, Mei YH, Li X, Yu DJ, Wang L, Chen X. Applying viscoplastic constitutive models to predict ratcheting behavior of sintered nanosilver lap-shear joint. Mech Mater 2014;72:61-71.

[6] Li X, Chen G, Wang L, Mei YH, Chen X, Lu GQ. Creep properties of low-temperature sintered nano-silver lap shear joints. Mat Sci Eng A-Struct 2013;579:108-13.

[7] Long X, Tang W, Feng Y, Chang C, Keer LM, Yao Y. Strain rate sensitivity of sintered silver nanoparticles using rate-jump indentation. Int J Mech Sci 2018;140:60-7.

[8] Ide E, Angata S, Hirose A, Kobayashi KF. Metal-metal bonding process using ag metallo-organic nanoparticles. Acta Mater 2005;53:2385-93.

[9] Long X, Tang W, Xia W, Wu Y, Ren L, Yao Y. Porosity and Young's modulus of pressure-less sintered silver nanoparticles. In: IEEE Electronics Packaging Technology Conference; 2017. p. 1-8.

[10] Zhao SY, Li X, Mei YH, Lu GQ. Novel interface material used in high power electronic die-attaching on bare Cu substrates. J Mater Sci-Mater El 2016;27:1-10.

[11] Paknejad SA, Mannan SH. Review of silver nanoparticle based die attach materials for high power/temperature applications. Microelectron Reliab 2017;70:1-11.

[12] Knoerr M, Schletz A, International conference on integrated power electronics systems, 2010, pp. 1-6.

[13] Lei TG, Calata JN, Lu GQ, Chen X, Luo S. Low-temperature sintering of nanoscale silver paste for attaching large-area $\left(>100 \mathrm{~mm}^{2}\right)$ chips. IEEE T Comp Pack Tech 2010;33:98-104.

[14] Zhang Z, Chen CT, Yang Y, Zhang H, Kim D, Sugahara T, Nagao S, Suganuma K. Low-temperature and pressureless sinter joining of $\mathrm{Cu}$ with micron/submicron $\mathrm{Ag}$ particle paste in air. J Alloy Compd 2019;780:435-42.

[15] Tan Y, Li X, Chen G, Mei Y, Chen X. Three-dimensional visualization of the crack-growth behavior of nano-silver joints during shear creep. J Electron Mater 2014;44:761-9.

[16] Zhang H, Chen C, Jiu J, Nagao S, Suganuma K. High-temperature reliability of low-temperature and pressureless micron $\mathrm{Ag}$ sintered joints for die attachment in high-power device. J Mater Sci-Mater El 2018;29:8854-62.

[17] Choe C, Noh S, Chen C, Ishina T, Nagao S, Suganuma K, Choe C, Noh S, Chen C, Ishina T, International conference on electronic packaging technology, 2017, pp. 1492-6.

[18] Chua ST, Siow KS, Jalar A, Proceedings of the IEEE/CPMT international electronics manufacturing technology symposium, 2015.

[19] Rmili W, Vivet N, Chupin S, Bihan TL, Quilliec GL, Richard C. Quantitative analysis of porosity and transport properties by FIB-SEM 3D imaging of a solder based sintered silver for a new microelectronic component. J Electron Mater 2016;45:2242-51

[20] Hay J, Agee P, Herbert E. Continuous stiffness measurement during instrumented indentation testing. Exp Techniq 2010;34:86-94.

[21] Oliver WC, Pharr GM. An improved technique for determining hardness and elastic modulus using load and displacement sensing indentation experiments. J Mater Res 1992:7:1564-83.

[22] Martin M, Troyon M. Fundamental relations used in nanoindentation: critical examination based on experimental measurements. J Mater Res 2002;17:2227-34.

[23] Tabor D. The hardness of metals. London: Oxford University Press; 1951.

[24] Carsley JE, Fisher A, Milligan WW, Aifantis EC. Mechanical behavior of a bulk nanostructured iron alloy. Metal Mater Trans A 1998;29:2261-71.

[25] Dean J, Wheeler JM, Clyne TW. Use of quasi-static nanoindentation data to obtain stress-strain characteristics for metallic materials. Acta Mater 2010;58:3613-23.

[26] Fu K, Li C, Zheng B, Tang Y, Wang H. On the determination of representative stress-strain relation of metallic materials using instrumented indentation. Mater Design 2015;65:989-94.

[27] Liu Z, Harsono E, Swaddiwudhipong S. Material characterization based on instrumented and simulated indentation tests. Int J Appl Mech 2009;1:61-84.

[28] Tho KK, Swaddiwudhipong S, Liu ZS, Zeng K. Simulation of instrumented indentation and material characterization. Mat Sci Eng A-Struct 2005;390:202-9.

[29] Long X, Feng Y, Yao Y. Cooling and annealing effect on indentation response of lead-free solder. Int J Appl Mech 2017;9:1750057.

[30] Leslie D, Dasgupta A, Morillo C. Viscoplastic properties of pressure-less sintered silver materials using indentation. Microelectron Reliab 2017;74:121-30.

[31] Ogasawara N, Chiba N, Xi C. Measuring the plastic properties of bulk materials by single indentation test. Scripta Mater 2006;54:65-70.

[32] Long X, Zhang X, Tang W, Wang S, Feng Y, Chang C. Calibration of a constitutive model from tension and nanoindentation for lead-free solder. Micromachines (Basel) 2018;9:1-13.

[33] Alkorta J, Martínez-Esnaola JM, Gil Sevillano J. Critical examination of strain-rate sensitivity measurement by nanoindentation methods: application to severely deformed niobium. Acta Mater 2008;56:884-93.

[34] Khrushchov MM, Berkovich ES. Methods of determining the hardness of very hard materials: the hardness of diamond. Ind Diamond Rev 1951;11:42-9.

[35] Chen X, Xiang Y, Vlassak JJ. Novel technique for measuring the mechanical properties of porous materials by nanoindentation. J Mater Res 2006;21:715-24.

[36] Haušild P, Materna A, Nohava J. On the identification of stress-strain relation by instrumented indentation with spherical indenter. Mater Design 2012;37:373-8.

[37] Howatson AM, Lund PG, Todd JD, Engineering tables and data, 1972. 